\title{
Protein Subcellular Localization Profiling of Breast Cancer Cells by Dissociable Antibody MicroArray (DAMA) Staining
}

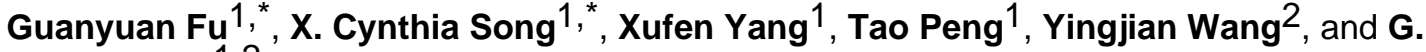 \\ Wayne Zhou ${ }^{1,2}$ \\ ${ }^{1}$ Department of Biological Sciences, Louisiana State University, Baton Rouge, LA 70803 \\ ${ }^{2}$ Hypromatrix Inc., 100 Barber Ave., Worcester, MA 01606
}

\begin{abstract}
We have developed Dissociable Antibody Microarray (DAMA) staining technology that provides a new approach to the global analysis of protein subcellular localization (SCL) in fixed cells. We have developed and optimized this technology for protein SCL profiling, generated ChipView, a program for management and analysis of molecular image database, and utilized the technique to identify proteins with unique SCL in breast cancer cell lines. We compared the SCL profiles of 325 proteins among nine different breast cell lines, and have identified one protein, Cyclin B1, with distinctively different SCLs between normal and cancer cell lines. With classic individual immunostaining, Cyclin B1 was confirmed to localize to the cytoplasm of seven breast cancer cell lines and in both cytoplasm and nuclei of two normal breast cell lines, and to have higher expression levels in the cancer cell lines tested.
\end{abstract}

\section{Keywords}

ChipView; cyclin B1; immunostaining; SCL profiling

\section{Introduction}

\begin{abstract}
Proteomics is an active and diverse field that uses different techniques to characterize and quantify proteome content, and to understand proteome structure and interactions, posttranslational modification, and biological function [1]. Among the techniques, protein microarrays have attracted attention for their potential use in high-throughput studies of protein function [2-7]. Microarrays have been used for studying protein expression profiles [8-10], protein-protein interactions [11,12], drug analysis [13,14], and for the diagnosis of diseases such as cancer, food allergies, and infection by pathogenic viruses and bacteria [1518]. Most protein microarrays use the capture microarray platform $[2,6,7]$ that captures a specific protein ligand from a mixture by an array of immobilized proteins on a solid support.
\end{abstract}

Profiling the localization of proteins in different subcellular compartments is as important as profiling protein expression and post-translational modification patterns. Knowledge of

Corresponding Author: G. Wayne Zhou, Department of Biological Sciences, Louisiana State University, Baton Rouge, LA 70803, zhouw2008@gmail.com, Fax: 508-302-0748.

These authors contributed equally

Conflict of interest statement

All authors declare no conflict interests. 
protein localization has important implications for understanding the activation state, interaction network, and biological function of the protein. For instance, nuclear localization of NF- $\kappa B$ is consistent with its activation through dissociation from I- $\kappa B$ proteins, which masks the NF- $\mathrm{KB}$ nuclear localization signal in the cytosolic complex $[19,20]$. One approach for studying protein localization is to separate the proteins of individual organelles into different cellular fractions. The components of each fraction can then be identified on a large scale with mass spectrometry [21,22]. An alternative approach is to visualize protein subcellular localization (SCL) in intact cells by fusing the interested proteins to fluorescent protein tags using techniques such as olgonucleotide-directed homologous recombination [23], mobile-element-based chromosomal tagging [24,25], or plasmid-based recombination cloning systems [26-28]. These techniques have been employed for large-scale analysis of protein SCLs in various organisms. Another widely accepted method for visualizing protein SCLs is immunostaining. However, the number of proteins that can be explored at one time is limited due to antibody cross-talk and the spectrum overlap of available labeling conjugates $[29,30]$.

We have developed a novel technology termed Dissociable Antibody Microarray (DAMA) staining [31] that combines the power of immunohistochemical staining with the parallel analytic capability of protein microarrays. This technology provides a new approach to the global analysis of both protein expression and SCLs in fixed cells. We have developed this technology and demonstrated its application in identifying potential biomarkers for breast cancer by profiling the expression of 312 proteins [32]. The unique advantage of this technology is its ability to determine the SCL profiles of hundreds of proteins from a single slide of samples. Here, we developed and optimized the technology for protein SCL profiling of hundreds of proteins in different breast cell lines. We also developed a computer program for the management and comparison of thousands of molecular images for proteins in different cell line samples and identified and validated proteins with unique SCLs between normal and cancer breast cell lines.

\section{Materials and methods}

\subsection{Preparation of antibody microarrays}

Four different antibody microarrays (Antibody Staining Array I - IV) were used for obtaining protein molecular images. Arrays I - III each contained 96 antibody spots, and Array IV contained 72 antibody spots. The antibodies were spotted by a robotic piezoelectric non-contact microarrayer on a $2 \times 2 \mathrm{~cm}$ membrane. Each spot contained $50 \mathrm{ng}$ antibody and had a diameter of $\sim 200 \mu \mathrm{m}$. The antibody lists for Staining Array I - IV are shown in supplementary Table S1. Antibodies were selected from Hypromatrix's collection (Worcester, MA). All antibodies were characterized and demonstrated to bind their targets.

\subsection{Cell culture}

Nine breast cell lines, MCF10A, Hs578Bst, MCF7, T-47D, ZR-75-1, MDA-MB-231, BT549, Hs578T, and MDA-MB-435S, were purchased from the American Type Culture Collection (ATCC, Manassas, VA). Cells were maintained and propagated as recommended by ATCC, and they were fixed at 90-95\% confluence for DAMA staining.

\subsection{Dissociable antibody microarray (DAMA) and individual classic staining}

Cells were fixed with $3.7 \%$ formaldehyde solution then permeabilized with $0.5 \%$ Triton X-100 and blocked with 10\% goat serum in PBS. For DAMA staining, antibody microarrays were placed over the cells and incubated at room temperature. A $0.2 \mathrm{~kg}$ weight, the optimal weight identified in earlier experiments, was placed on top of the staining apparatus for 2 hours. For classic staining, individual primary antibodies diluted 1:500 in PBS were 
delivered to the surface of the cells followed by incubation at room temperature for 2 hours. Bound antibodies were detected by Alexa Fluor 488-conjugated secondary antibodies (both goat-anti-rabbit and goat-anti-mouse for DAMA staining, and only one used for individual staining depending on the source of the primary antibodies) for 30 minutes. Cells were then stained with DAPI for 5 minutes before being mounted on slides for fluorescent microscopy.

\subsection{Fluorescent microscopy}

Fluorescent microscopy were performed with an imaging system consisting of Leica DM RXA2 upright microscope (Leica Microsystem, Bannockburn, IL), a SensiCam 12-bit CCD camera (The Cooke Corporation, Romulus, MI), a motorized stage and the SlideBook 4.1 software (Intelligent Imaging Innovation, Santa Monica, CA). For DAMA staining, the molecular images of 96 or 72 proteins were obtained automatically from a single cell culture coverslip with the multi-well capture package of SlideBook. Protein molecular images from individual staining were obtained with the standard capture package.

\section{Results}

We have previously demonstrated that the molecular images of proteins obtained using DAMA staining have similar quality to those obtained by classic immunostaining [31]. The goal of this work was to develop the technique for protein SCL profiling and to demonstrate its application in identifying proteins with different SCLs between normal and cancer breast cell lines. The experimental procedure included the following steps: determination of the protein SCL profiles by DAMA staining, database construction and data analysis by the ChipView program, identification of proteins with altered SCLs in normal and cancer breast cells, and evaluation of the identified proteins by individual classic immunostaining.

\subsection{Determination of the SCL profiles of 360 proteins in 9 different breast cell lines by DAMA staining}

After determining the optimal conditions (Fig S1), protein SCL profiles of nine different cell lines from human mammary glands were obtained by DAMA staining. The nine breast cell lines include two normal lines, Hs578Bst and MCF10A, and seven carcinoma-derived cell lines, T-47D, MCF7, ZR-75-1, MDA-MB-231, BT549, Hs578T, and MDA-MB-435S. The cells were grown on standard coverslips to $\sim 90 \%$ confluence, and fixed and permeabilized by a standard protocol. Three hundred sixty antibodies were delivered to the fixed cells using four different antibody arrays (Antibody Array I, II, III and IV). Bound antibodies were then detected using fluorescent-conjugated secondary antibodies. Images of the 360 proteins, each repeated five times, were obtained in the green channel with the DAPI stained images in blue channel, by using an automatic imaging system (microscope equipped with a computer-controlled motorized stage). A total of approximately 16,200 molecular images was obtained and stored for database construction and analysis.

\subsection{Database management and image analysis by ChipView}

ChipView, a database-based GUI program, was developed to manage and analyze the large amount of molecular images obtained above (Fig S2). An image database was established and expanded by importing original images of the 360 proteins in the nine breast cell lines. The database holds images and related sample information such as array type, sample name, experimental conditions, and SCL patterns, in interrelated tables. This relational database design facilitates the search and presentation of images by their biological or experimental information. The program can assign a custom-defined SCL code for each protein and can compare the molecular images of every protein in all samples, as discussed below. The program can also select a representative molecular image for all 360 proteins in one cell line and stitch them together as a chipview picture, which represents the SCL profile of those 
proteins in the cell line. The chipview pictures of 360 proteins in cell lines Hs578Bst, a normal breast cell line, and T-47D, a breast cancer cell line, are shown in Fig 1. The chipviews for the rest of the cell lines are shown in Fig S3. In total, 3,240 representative molecular images were incorporated in the nine chipviews.

\subsection{Initial validation of the protein SCL profiles obtained by DAMA staining}

The protein SCL in different breast cell lines was assigned by visually assessing each molecular image with the ChipView program using the following characterization. Based on whether the green channel signal of the captured image overlapped with its blue channel signal of DAPI staining, the SCL of the studied protein in each cell was defined as nuclear, cytoplasmic, or both, and was assigned as N, C, or B, respectively. The SCL of a given protein in a molecular image was assigned with the most dominant SCL pattern of that protein in the image. For those proteins with poor quality images due to low expression, out of focus, without cells in the captured images, or proteins with uncertain images (no single dominant SCL pattern in the image), a code " $U$ " was assigned.

To examine the accuracy of the SCL profiles assigned from the DAMA staining images, the SCL profiles of 96 proteins, stained with Antibody Array I, were compared between two independent experiments for cell lines T47D and MCF10A. Molecular images of the 96 proteins were captured using the automatic imaging system with a $63 \times$ objective lens. The SCLs in the two independent image datasets were assigned and compared. In the T47D cell line sample, 73 proteins have identical SCL assignments between the two image sets and 20 proteins were assigned as $U$ from one of the image sets. Only three proteins were assigned differently between the two image sets. In the MCF10A cell line sample, 64 proteins have identical SCL assignments between the two independent image sets and 23 proteins were assigned as "U" from one of the image datasets. Only nine proteins were assigned differently between the two independent sets. The discrepancy and large number of " $U$ " designations in at least one image set were a result of the systematic focus deviation and heterogeneity of cell status in the experiment. Despite this, the above results suggested that reproducible SCL profiles could be obtained from the DAMA staining experiments.

\subsection{Comparison of the SCL profiles from image datasets using different objective lenses}

To evaluate the accuracy of the SCL profiles obtained from the DAMA staining, all the images for nine different cell lines were captured independently by using a both $20 \times$ objective lens and a $63 \times$ objective lens. The SCLs in each image of the $20 \times$ dataset and the $63 \times$ dataset were assigned by visual inspection using the ChipView program. The assigned codes from two independent datasets were compared (Fig 3a). The consistency of SCL assignments from two image datasets ranged from $91 \%$ to $100 \%$ in nine different cell lines, with an average of $95 \%$ consistency. For the SCL assignment of all 3,240 proteins in nine cell lines, about $70 \%$ were assigned the identical code from two datasets (codes other than "U"), and $25 \%$ were assigned "U" in at least one dataset, due to low quality images in either image set. These results suggest that the SCL profiles assigned from the $63 \times$ image dataset were consistent with those assigned from the $20 \times$ image dataset.

\subsection{SCL profiles of $\mathbf{3 6 0}$ proteins from nine different breast cell lines}

The SCL profiles of 360 proteins from nine different breast cell lines are summarized in Fig 2. Each colored bar represents the SCL of a specific protein in a specific cell line. Red, green, and yellow colored bars represent protein localization to the nuclei $(\mathrm{N})$, cytoplasm (C), or both (B), respectively, as assigned from their DAMA staining images. The final SCL assignments were determined by incorporating the assignments from both the $20 \times$ image dataset and the $63 \times$ image dataset (Fig 2). Among these assignments, an average of $13 \%$ of the proteins were assigned as "N", $61 \%$ were assigned as "C", and $16 \%$ were assigned as 
"B" in the seven cancer cell lines. The average percent of proteins assigned to " $N$ ", "C", or

"B" in the two normal cell lines was 30\%,10\%, and 48\%, respectively (Fig $3 b$ ). Of the 360

proteins, one protein, Cyclin B1 (I-B06), was identified as having distinctively different

localization between the normal and cancer cell lines (Fig 3c). This protein distributed

evenly within most cells from both normal cell lines and localized mainly in the cytoplasm

of all seven cancer cell lines.

\subsection{Validation of the assigned protein SCLs by individual immunostaining}

To evaluate the accuracy of assigned SCL profiles from the DAMA staining images, the images of 31 proteins were obtained by individual classic immunostaining and were compared with their images obtained from the DAMA staining (Table S2). Of the 31 proteins, the SCL assignments of four proteins were confirmed in all eight cell lines examined by individual immunostaining, 10 proteins were confirmed in seven of the cell lines, and 15 proteins were confirmed in six of the cell lines. One protein was confirmed in five out of eight cell lines examined. The inconsistency is due mainly to the assignment of "C" from one image and "B" in the other images, because of poor quality DAMA staining of images. Therefore, among the SCL codes assigned for the 248 molecular images obtained from DAMA staining, 201 have been confirmed by individual immunostaining.

\subsection{Cyclin B1 was shown to be distributed to different locations between normal and cancer cell lines}

From the comparison of SCL profiles, Cyclin B1 was identified as having a different subcellular distribution between normal and cancer cell lines. The observed difference in SCL of Cyclin B1 in nine different cell lines was examined and confirmed by individual immunostaining (Fig 4a). Cyclin B1 showed predominantly cytoplasmic localization in the seven cancer cell lines, but distributed nearly evenly to both nuclei and cytoplasm in the majority of the two normal cell lines. In addition, by comparing the intensities of the immunostained Cyclin B1 images, the staining level in normal cell lines was much lower than that in the cancer cell lines, which suggests possible lower expression of Cyclin B1 in normal cell lines. To test this hypothesis, the expression level of Cyclin B1 in the nine cell lines was examined by western blot analysis. Indeed, the expression level of Cyclin B1 was confirmed to be much lower in normal cell lines than in the cancer cell lines (Fig 4b). The expression of Cyclin B1 was nearly undetectable in the two normal cell lines. The expression of Cyclin B1 varied in the seven cancer cell lines, but each was higher than that in normal cell lines.

\section{Discussion}

Dissociable antibody microarray (DAMA) staining is a novel protein microarray platform that can be used to study both protein expression and SCL profiles in a high throughput fashion. In this report, molecular images of 360 proteins from nine breast cell lines were obtained by using DAMA staining technology and were analyzed with ChipView, a program for database management and analysis. The SCL profiles of these proteins in the nine cell lines were determined and reported in a single color code profile. The SCL patterns of the 360 proteins were examined and compared between normal and cancer cell lines. One protein, cyclin B1, was identified as being distributed evenly throughout the cell and with low expression in normal cell lines, and localized mainly to the cytoplasm with higher expression in cancer cell lines.

Cyclin B1 is a key regulator of cell cycle progression that accumulates predominantly in the cytoplasm during S-G2 phase, redistributes to the nucleus during mitosis, and is then destroyed during the metaphase-anaphase transition in breast lesions [33]. Therefore, 
differential cyclin B1 localization can be observed: nucleus, cytoplasm, or whole cell. In both normal and carcinoma breast tissues, cyclin B1 is commonly localized to the cytoplasm. Our results are partially consistent with previous breast tissue studies in that all seven cancer cell lines tested showed a dominant cytoplasmic cyclin B1 localization, although nucleus and whole cell localizations were also observed. In contrast, the most prevalent localization of cyclin B1 in the two normal cell lines tested was throughout the whole cell. One possible reason may be that most cells of the two normal cell lines were in G1 phase, during which only basal expression of cyclin B1 was present due to the degradation of cyclin B1 in M phase. The fact that the expression of cyclin B1 in normal cell lines was nearly undetectable by western blot (Fig $4 \mathrm{~b}$ ) supported this assumption. Overexpression of cyclin B1 has been reported in breast cancer cell lines [34,35] and breast carcinomas [33]. Consistent with these findings, our results also demonstrate elevated expression of cyclin B1 in breast cancer cell lines relative to normal breast cell lines. Indeed, the expression and SCL of cyclin B1 have been proposed for the prognosis of breast carcinomas [36-39].

In our current study, we used very strict criteria to identify proteins with differential localization between the normal and cancer breast cell lines. When a less stringent criterion was applied, six more proteins, Flt3/2 (I-C06), NF2 (I-E11), Vav (I-H09), CIAP2 (II-E05), Syntaxin (IV-C09), SOCS1 (IV-E05), were identified with the altered SCL patterns between the normal and cancer breast cell lines. From the DAMA staining images, these proteins were assigned with distinct SCL codes between the normal and six out of the seven cancer cell lines, with the seventh assigned as "U". The SCLs of these six proteins were then validated with individual immunostaining, especially for the cell line with a " $U$ " assignment (Table S2). The SCL pattern in the "U"-assigned cell line was assigned differently from other six cancer cell lines by individual immunostaining for five proteins, Flt3/2, SOCS1, NF2, CIAP2 and Vav. These results disqualified them as the proteins having the altered SCL patterns between the normal and cancer cell lines. The SCL pattern of Syntaxin was replicated in all seven cancer cell lines, but was assigned differently in the tested normal cell line.

Other patterns of SCL difference between the normal and cancer breast cell lines could also be observed from the DAMA staining results. For example, seven proteins, Smad1 (I-G10), A1 (II-A2), Bin1 (II-C1), Syntaxin (IV-C09), TRAF6 (IV-D06), P-Selectin (IV-E1), ZO-1 (IV-F11), were assigned to be localized in the nuclei of the normal cell lines, but were assigned with patterns other than nucleus in the cancer cell lines (i.e. "C", "B" or "U"). Among these proteins, Smad1 and Syntaxin were assigned as " $U$ " in one cancer cell line, and A1, Bin1, P-Selectin, and ZO-1 were assigned as "U" in two cancer cell lines. In their individual immunostaining images, all seven proteins were found to have weak signal in both normal breast cell lines. Therefore, their observed nuclear localization patterns in the normal cell lines could be due to the non-specific background staining, which was supported by the images obtained at the positions without primary antibodies immobilized in the same DAMA staining slides. Those images indeed appear with the nucleus pattern in both normal cell lines. The non-specific background staining could also explain the various results for Syntaxin obtained from different staining experiments in normal cell lines.

Recently, several databases, such as LOCATE, a mammalian protein subcellular localization database, and Human Protein Atlas (HPA), have been developed for the expression and spatial distribution of proteins in different tissue and cell line samples [40-42]. We have examined the SCLs of the 360 proteins investigated in this study by using HPA, and found the SCL patterns of 278 proteins from this database. Among them, 47 proteins exhibit single SCL patterns in the tissue stained positively, with 14 localized in nucleus, 32 in cytoplasm and 1 on membrane. Of the 47 proteins, 18 have no experimental SCL information available 
in the antibody supplier's database, and 7 were localized in different SCL from those identified from the HPA database. Combining the information together, 22 out of 360 proteins exhibit a single SCL pattern from both databases.

The DAMA staining technology is an alternative approach to determine the SCL profiles. Our results show that 20 proteins have the same SCL patterns in all nine tested cell lines. Of the 20 proteins, 9 have the same SCL patterns as those identified from the above databases, and 11 have different SCL patterns. The difference in SCL patterns could be due to the different samples included in different databases. The HPA database includes both the tissue and cell line samples from a variety of origins, and only human breast cell line samples were used in the DAMA staining experiment.

During SCL assignment of images obtained from DAMA staining, about 10\% of the proteins were coded " $U$ " in their images. The images from DAMA staining were captured with an automatic imaging system, which captures the image with automatic focus and automatic exposure time based on staining intensity. To improve the image quality of some proteins, more robust algorithms for automatic focus could be employed. This will require a wider range of $z$ length to include the best focus in the search range. In this work, we determined the SCL of proteins by visual inspection of their molecular images against DAPI staining one at a time. This manual annotation of images is labor intensive and tends to involve annotator's subjectivity, especially when certain patterns are expected with previous knowledge. Therefore, new algorithms for automatic assignment of protein localization patterns cell by cell using colocalization information of the protein with the organelle markers will be developed. A pilot study (Fu et al., unpublished data) using colocalization information of particular protein and the nuclear marker DAPI in 2-D images has shown the feasibility of such a strategy.

The DAMA staining technique is a combination of microarray technology and classic immunostaining. To our knowledge, this is the first technique that can determine the SCLs of hundreds of proteins from a single experimental sample. A microarray with 400 antibodies has been developed, and one with 1,000 antibodies is also achievable. We have demonstrated the application of the technique for the systematic study of protein SCL profiles, and identified and confirmed one protein with altered SCL and expression levels between normal and cancer breast cell lines. The technique could also be a powerful tool for studying the expression and SCL profiles and trafficking of hundreds or thousands of proteins following signal stimulation.

\section{Supplementary Material}

Refer to Web version on PubMed Central for supplementary material.

\section{Abbreviation}

DAMA dissociable antibody microarray

SCL subcellular localization

\section{Acknowledgments}

We thank Chandran Komma, Ling Ying, and Wei Wang for their help on different stages of the project. This work was supported by National Institutes of Health grant CA110047 and a grant from the Susan G Komen Breast Cancer Foundation. 


\section{References}

1. Falk R, Ramstrom M, Stahl S, Hober S. Approaches for systematic proteome exploration. Biomol Eng. 2007; 24:155-168. [PubMed: 17376740]

2. Bertone P, Snyder M. Advances in functional protein microarray technology. FEBS J. 2005; 272:5400-5411. [PubMed: 16262682]

3. de Wildt RM, Mundy CR, Gorick BD, Tomlinson IM. Antibody arrays for high-throughput screening of antibody-antigen interactions. Nat Biotechnol. 2000; 18:989-994. [PubMed: 10973222]

4. Emili AQ, Cagney G. Large-scale functional analysis using peptide or protein arrays. Nat Biotechnol. 2000; 18:393-397. [PubMed: 10748518]

5. Hall DA, Ptacek J, Snyder M. Protein microarray technology. Mech Ageing Dev. 2007; 128:161167. [PubMed: 17126887]

6. Kodadek T. Protein microarrays: prospects and problems. Chem Biol. 2001; 8:105-115. [PubMed: 11251285]

7. MacBeath G. Protein microarrays and proteomics. Nat Genet. 2002; 32 Suppl:526-532. [PubMed: 12454649]

8. Knezevic V, Leethanakul C, Bichsel VE, Worth JM, et al. Proteomic profiling of the cancer microenvironment by antibody arrays. Proteomics. 2001; 1:1271-1278. [PubMed: 11721638]

9. Schweitzer B, Roberts S, Grimwade B, Shao W, et al. Multiplexed protein profiling on microarrays by rolling-circle amplification. Nat Biotechnol. 2002; 20:359-365. [PubMed: 11923841]

10. Sreekumar A, Nyati MK, Varambally S, Barrette TR, et al. Profiling of cancer cells using protein microarrays: discovery of novel radiation-regulated proteins. Cancer Res. 2001; 61:7585-7593. [PubMed: 11606398]

11. Pawlak M, Schick E, Bopp MA, Schneider MJ, et al. Zeptosens' protein microarrays: a novel high performance microarray platform for low abundance protein analysis. Proteomics. 2002; 2:383393. [PubMed: 12164697]

12. Uetz P, Giot L, Cagney G, Mansfield TA, et al. A comprehensive analysis of protein-protein interactions in Saccharomyces cerevisiae. Nature. 2000; 403:623-627. [PubMed: 10688190]

13. Huang RP, Yang W, Yang D, Flowers L, et al. The promise of cytokine antibody arrays in the drug discovery process. Expert Opin Ther Targets. 2005; 9:601-615. [PubMed: 15948677]

14. Zhu H, Bilgin M, Bangham R, Hall D, et al. Global analysis of protein activities using proteome chips. Science. 2001; 293:2101-2105. [PubMed: 11474067]

15. Bacarese-Hamilton T, Bistoni F, Crisanti A. Protein microarrays: from serodiagnosis to whole proteome scale analysis of the immune response against pathogenic microorganisms. Biotechniques. 2002 Suppl:24-29. [PubMed: 12514926]

16. Mezzasoma L, Bacarese-Hamilton T, Di Cristina M, Rossi R, et al. Antigen microarrays for serodiagnosis of infectious diseases. Clin Chem. 2002; 48:121-130. [PubMed: 11751547]

17. Robinson WH, DiGennaro C, Hueber W, Haab BB, et al. Autoantigen microarrays for multiplex characterization of autoantibody responses. Nat Med. 2002; 8:295-301. [PubMed: 11875502]

18. Woodbury RL, Varnum SM, Zangar RC. Elevated HGF levels in sera from breast cancer patients detected using a protein microarray ELISA. J Proteome Res. 2002; 1:233-237. [PubMed: 12645900]

19. Baeuerle PA, Henkel T. Function and activation of NF-kappa B in the immune system. Annu Rev Immunol. 1994; 12:141-179. [PubMed: 8011280]

20. Siebenlist U, Franzoso G, Brown K. Structure, regulation and function of NF-kappa B. Annu Rev Cell Biol. 1994; 10:405-455. [PubMed: 7888182]

21. Dreger M. Proteome analysis at the level of subcellular structures. European Journal of Biochemistry. 2003; 270:589-599. [PubMed: 12581199]

22. Tan DJL, Arias AM. High-throughput localization of organelle proteins by mass spectrometry: a quantum leap for cell biology. Bioessays. 2006; 28:780-784. [PubMed: 16927390]

23. Huh WK, Falvo JV, Gerke LC, Carroll AS, et al. Global analysis of protein localization in budding yeast. Nature. 2003; 425:686-691. [PubMed: 14562095] 
24. Jarvik JW, Fisher GW, Shi C, Hennen L, et al. In vivo functional proteomics: mammalian genome annotation using CD-tagging. Biotechniques. 2002; 33:852-854. 856, 858-860 passim. [PubMed: 12398194]

25. Morin X, Daneman R, Zavortink M, Chia W. A protein trap strategy to detect GFP-tagged proteins expressed from their endogenous loci in Drosophila. Proc Natl Acad Sci U S A. 2001; 98:1505015055. [PubMed: 11742088]

26. Ding DQ, Tomita Y, Yamamoto A, Chikashige Y, et al. Large-scale screening of intracellular protein localization in living fission yeast cells by the use of a GFP-fusion genomic DNA library. Genes Cells. 2000; 5:169-190. [PubMed: 10759889]

27. Escobar NM, Haupt S, Thow G, Boevink P, et al. High-throughput viral expression of cDNAgreen fluorescent protein fusions reveals novel subcellular addresses and identifies unique proteins that interact with plasmodesmata. Plant Cell. 2003; 15:1507-1523. [PubMed: 12837943]

28. Simpson JC, Wellenreuther R, Poustka A, Pepperkok R, Wiemann S. Systematic subcellular localization of novel proteins identified by large-scale cDNA sequencing. EMBO Rep. 2000; 1:287-292. [PubMed: 11256614]

29. Schieker M, Pautke C, Reitz K, Hemraj I, et al. The use of four-colour immunofluorescence techniques to identify mesenchymal stem cells. Journal of Anatomy. 2004; 204:133-139. [PubMed: 15032920]

30. Staines WA, Meister B, Melander T, Nagy JI, Hokfelt T. Three-color immunofluorescence histochemistry allowing triple labeling within a single section. J Histochem Cytochem. 1988; 36:145-151. [PubMed: 2891745]

31. Wang Y. Immunostaining with dissociable antibody microarrays. Proteomics. 2004; 4:20-26. [PubMed: 14730668]

32. Song XC, Fu G, Yang X, Jiang Z, et al. Protein expression profiling of breast cancer cells by dissociable antibody microarray (DAMA) staining. Mol Cell Proteomics. 2008; 7:163-169. [PubMed: 17934210]

33. Kawamoto H, Koizumi H, Uchikoshi T. Expression of the G2-M checkpoint regulators cyclin B1 and cdc2 in nonmalignant and malignant human breast lesions: immunocytochemical and quantitative image analyses. Am J Pathol. 1997; 150:15-23. [PubMed: 9006317]

34. Barrett KL, Demiranda D, Katula KS. Cyclin b1 promoter activity and functional cdk1 complex formation in G1 phase of human breast cancer cells. Cell Biol Int. 2002; 26:19-28. [PubMed: 11779217]

35. Androic I, Kramer A, Yan R, Rodel F, et al. Targeting cyclin B1 inhibits proliferation and sensitizes breast cancer cells to taxol. BMC Cancer. 2008; 8:391. [PubMed: 19113992]

36. Kuhling H, Alm P, Olsson H, Ferno M, et al. Expression of cyclins E, A, and B, and prognosis in lymph node-negative breast cancer. J Pathol. 2003; 199:424-431. [PubMed: 12635132]

37. Rudolph P, Kuhling H, Alm P, Ferno M, et al. Differential prognostic impact of the cyclins E and $\mathrm{B}$ in premenopausal and postmenopausal women with lymph node-negative breast cancer. Int J Cancer. 2003; 105:674-680. [PubMed: 12740917]

38. Suzuki T, Urano T, Miki Y, Moriya T, et al. Nuclear cyclin B1 in human breast carcinoma as a potent prognostic factor. Cancer Sci. 2007; 98:644-651. [PubMed: 17359284]

39. Winters ZE, Hunt NC, Bradburn MJ, Royds JA, et al. Subcellular localisation of cyclin B, Cdc2 and p21(WAF1/CIP1) in breast cancer. association with prognosis. Eur J Cancer. 2001; 37:24052412. [PubMed: 11720835]

40. Uhlen M, Bjorling E, Agaton C, Szigyarto CA, et al. A human protein atlas for normal and cancer tissues based on antibody proteomics. Mol Cell Proteomics. 2005; 4:1920-1932. [PubMed: 16127175]

41. Berglund L, Bjorling E, Oksvold P, Fagerberg L, et al. A genecentric Human Protein Atlas for expression profiles based on antibodies. Mol Cell Proteomics. 2008; 7:2019-2027. [PubMed: 18669619]

42. Sprenger J, Lynn Fink J, Karunaratne S, Hanson K, et al. LOCATE: a mammalian protein subcellular localization database. Nucleic Acids Res. 2008; 36:D230-D233. [PubMed: 17986452] 

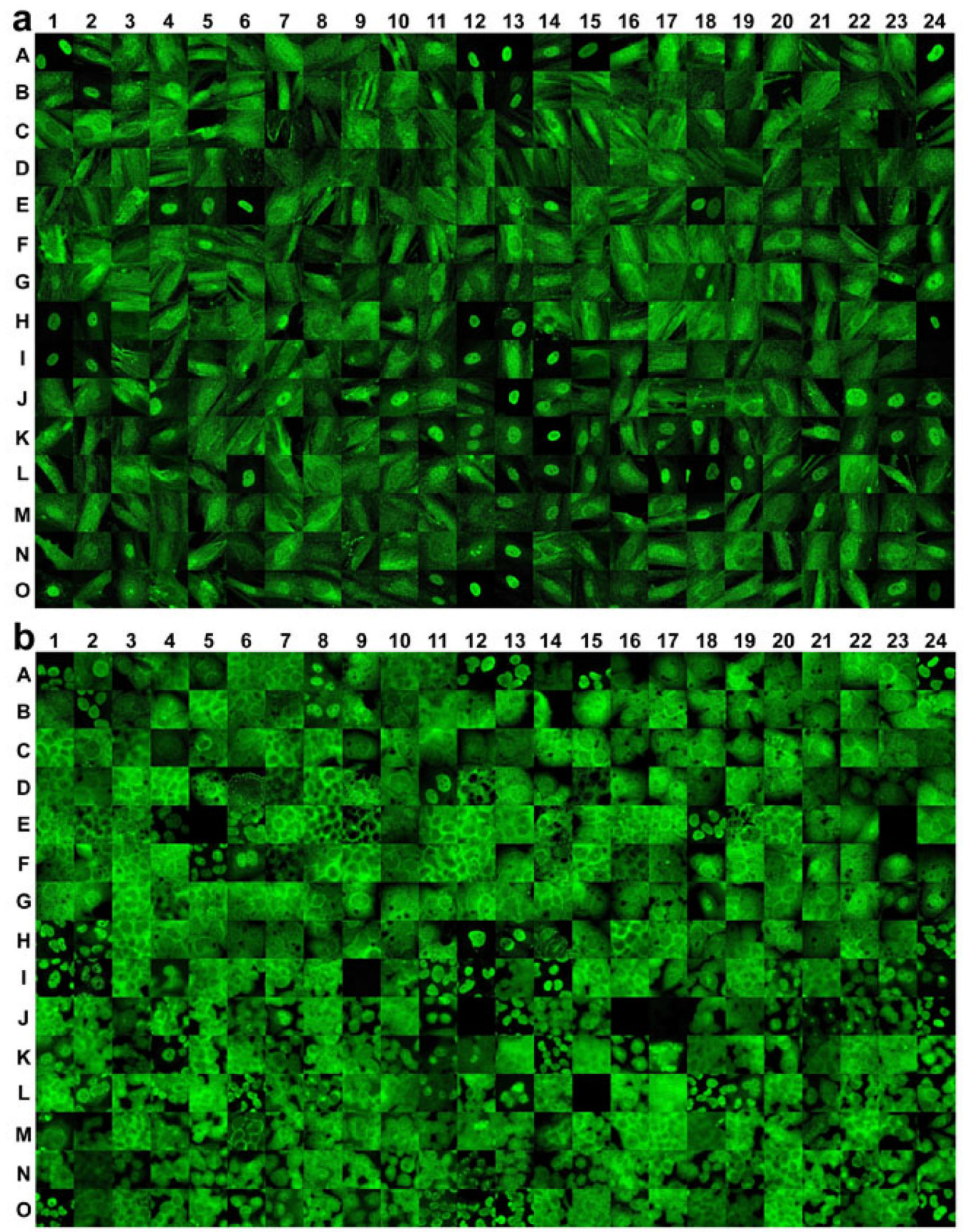

Fig 1. Chipview pictures of representative molecular images of 360 proteins in one benign breast cell line (Hs578Bst) (a) and in one breast cancer cell line (T-47D) (b) obtained by the DAMA staining technique

The molecular images of 360 proteins obtained from DAMA staining were first imported to a database. Representative images were selected to construct the picture by using the ChipView program (Fig S2) and arranged in the same layout as the antibody list shown in Table S1. Each image represents the image of the corresponding protein in the sample tested. For those proteins whose molecular images at $63 \times$ magnification were not readable, black images were used. 


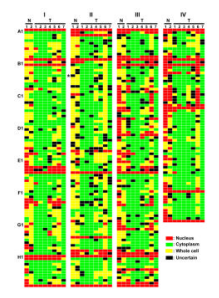

Fig 2. SCL profiles of 360 proteins in two benign and seven carcinoma derived cell lines SCL profiles are presented with a custom-defined color code scheme. Red indicates a protein is localized to the nuclei, green indicates a protein is localized to the cytoplasm, yellow indicates a protein is localized both to nuclei and in cytoplasm, and black represents an uncertain SCL for the protein. The final SCL assignments were determined by incorporating the assignments from both the $20 \times$ image dataset and the $63 \times$ image dataset. If the assignment of images from two magnification datasets was same, the same code was used. If the two are different and both have a "non- $U$ " code $(\mathrm{N}, \mathrm{C}, \mathrm{B})$, then a "U" is given, and if images from any of the two sets was assigned "U", then the code from the other set was used in the final assignment. Each column represents the SCL codes of the proteins in the same cell line, and each row represents the SCL codes of the same protein in different cell line samples. Those proteins identified with different SCLs between the benign and cancer cell lines were marked by asterisks at the end of the row. The cell lines corresponding to the labels on the figure are: N1, MCF10A; N2, Hs578Bst; T1, T-47D; T2, MCF7; T3, ZR-75-1; T4, MDA-MB-231; T5, BT549; T6, Hs578T; T7, MDA-MB-435S. 


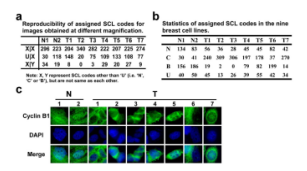

Fig 3. Summary of SCL profiles of 360 proteins in nine breast cell lines, as assigned from the images obtained with the DAMA staining technology

a) Analysis of the reproducibility of the assigned SCL codes. Case $1(\mathrm{X} \mid \mathrm{X})$ represents those proteins which have the same assignments (not "U") in both $20 \times$ and $63 \times$ images, case 2 (U) $\mathrm{X})$ represents the proteins which have an uncertain assignment ("U") in either $20 \times$ images or $63 \times$ images, and case $3(\mathrm{X} \mid \mathrm{Y})$, proteins which have certain assignments in both the $20 \times$ and $63 \times$ images, but are different from one another. b) Summary of the statistics of the assigned SCL distribution in nine breast cell lines. c) The molecular images of Cyclin B1 obtained from the DAMA staining in the nine breast cell lines possessing different SCL codes in benign and carcinoma derived cell lines. Labels for cell lines follow the same routine as in Figure 2. 


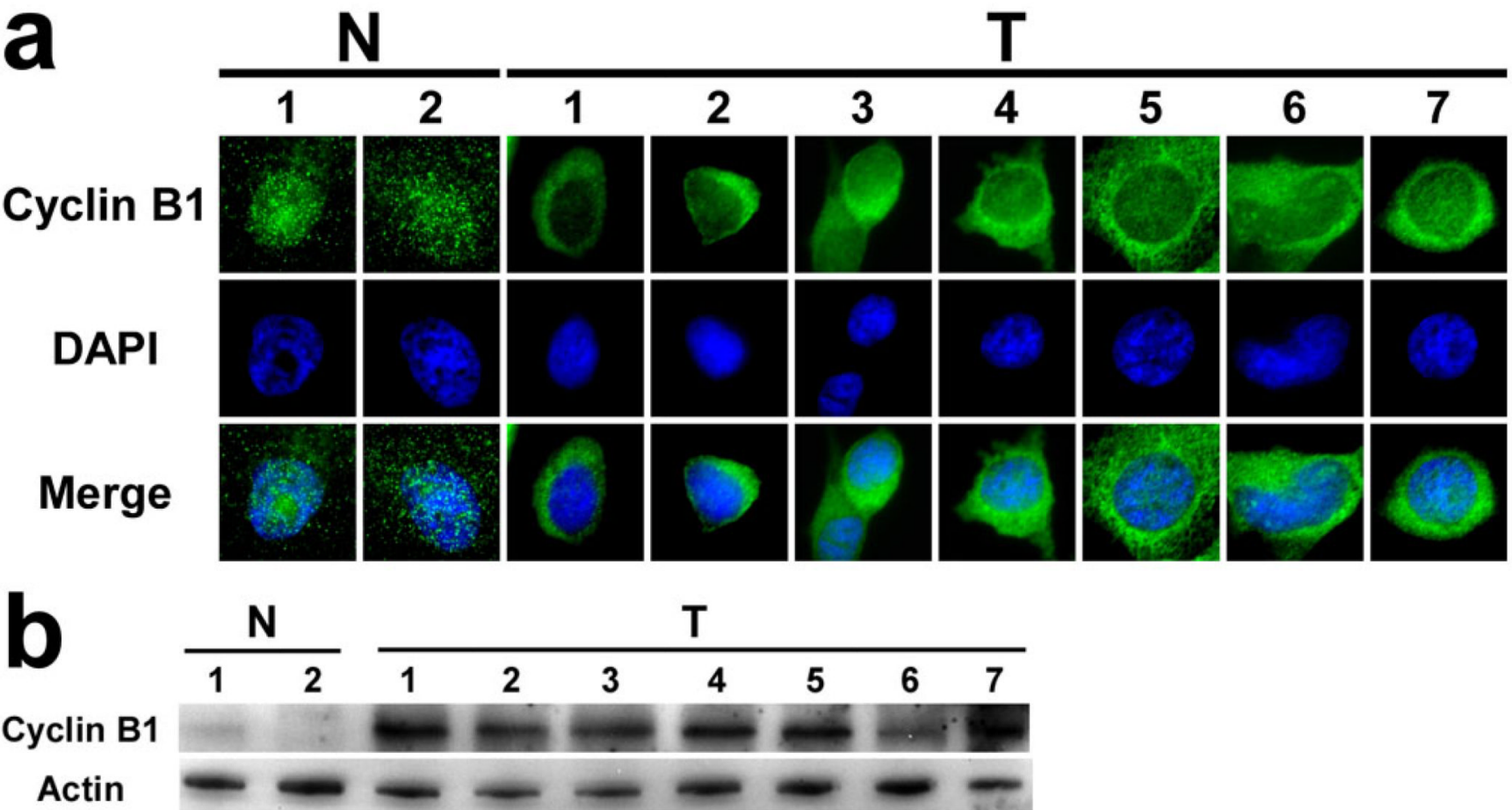

Fig 4. Cycle B1 was confirmed to have altered SCL and expression in benign and carcinomaderived breast cell lines

a) Individual immunostaining confirmed Cyclin B1 to have altered SCL in benign and carcinoma derived breast cell lines as determined by DAMA staining (Fig. 3c). b) Expression of Cyclin B1 in the nine cell lines determined by western blot. Labels for cell lines follow the same routine as in Figure 2. 